\title{
TOPOLOGICAL MODELS OF FINITE TYPE FOR TREE ALMOST AUTOMORPHISM GROUPS
}

\author{
ROMAN SAUER AND WERNER THUMANN
}

\begin{abstract}
We show that tree almost automorphism groups, including Neretin groups, satisfy the analogue of the $F_{\infty}$-finiteness condition in the world of totally disconnected groups: They possess a cellular action on a contractible cellular complex such that the stabilizers are open and compact and the restriction of the action on each $n$-skeleton is cocompact.
\end{abstract}

\section{INTRODUCTION}

The Neretin groups $\mathcal{N}_{q}$ - introduced by Neretin as $p$-adic analogues of the diffeomorphism group of the circle - are remarkable locally compact groups. They are simple, hence unimodular [14]. But they do not possess any lattice 2] (the first simple such example), and they are compactly presented [16.

The Neretin groups lie in the larger family of tree almost automorphism groups $\mathcal{A}_{q r}^{D}$ (Definition 4.3). It is $\mathcal{N}_{q} \cong \mathcal{A}_{q 2}^{\mathrm{Sym}(q)}$. Compact presentability [16] of the groups $\mathcal{A}_{q r}^{D}$ (denoted by $\operatorname{AAut}_{D}\left(\mathcal{T}_{q, r}\right)$ in [16]) also hold true here, and simplicity results were obtained in 8 .

The Higman-Thompson groups $F_{q r}$ and $V_{q r}$ embed into $\mathcal{A}_{q r}^{D}$ discretely and densely, respectively (Remark 4.4). For $q=2$ and $r=1$ these are the famous Thompson groups $F$ and $V$. Thompson's group $F$ was the first known torsionfree group that satisfies the finiteness condition of being of type $F_{\infty}$ and has infinite cohomological dimension [7.

Our goal is to show that the tree almost automorphism groups $\mathcal{A}_{q r}^{D}$ satisfy a similar $F_{\infty}$-property in the context of totally disconnected groups.

A discrete group $\Gamma$ is said to be of type $F_{\infty}$ if it acts freely on a contractible $\mathrm{CW}$ complex $X$ by cell permuting homeomorphisms such that there are only finitely many $\Gamma$-orbits of $n$-cells for every $n$; in common terminology, recalled in Subsection 2.1 $X$ is a contractible free $\Gamma$-CW-complex of finite type. Non-discrete totally disconnected groups cannot act freely on $\mathrm{CW}$-complexes by cell permuting homeomorphisms. Thus we turn to an equivalent description of type $F_{\infty}$ of discrete groups which leads to the right notion in the totally disconnected setting.

Proposition 1.1 (18, Lemma 4.1]). A discrete group $\Gamma$ is of type $F_{\infty}$ if and only if there is a contractible proper $\Gamma-C W$-complex of finite type.

Here, properness is equivalent to finiteness of stabilizers. For a totally disconnected group $G$ we consider $G$-CW-complexes which are smooth, i.e. stabilizers are open, and proper, i.e. stabilizers are compact. A smooth $G$-CW-complex is, in particular, a CW-complex when discarding the group action (Remark 2.2).

Definition 1.2. Let $G$ be a totally disconnected group. A topological model of $G$ is a contractible proper smooth $G$-CW-complex. We say that $G$ is of type $F_{\infty}$ if $G$ admits a topological model of finite type.

2010 Mathematics Subject Classification. Primary 55R35; Secondary 20E08, 22 D05.

Key words and phrases. Tree almost automorphism group, Neretin group, finiteness properties. 
Remark 1.3. Type $F_{\infty}$ implies being compactly generated and compactly presented (Proposition 2.5).

The theory of finiteness conditions for totally disconnected or locally compact groups is still less developed than the one for discrete groups. The notion of compact presentation was first introduced in 1964 by Kneser [15] which we learned from the forthcoming book [10. Abels and Tiemeyer introduced finiteness conditions for arbitrary locally compact groups in terms of group cohomology [1. The relation of their property $C_{\infty}$ to the above $F_{\infty}$-property will be discussed in forthcoming work of the authors of this paper. A homological setup over the rationals for finiteness conditions of totally disconnected groups was recently developed in 9 .

We are ready to state our main result which is proved in Subsection 4.5. It generalizes the result of Le Boudec [16] on compact presentability.

Theorem 1.4. The groups $\mathcal{A}_{q r}^{D}$ are of type $F_{\infty}$.

The ideas in Subsections 4.2, 4.3 and 4.4 are inspired by the finiteness results for discrete Thompson-like groups. An interesting feature of our approach is that we simultaneously prove finiteness results for non-discrete totally disconnected groups and discrete groups. Indeed, in the case $D=\{1\}, q=2, r=1$ Theorem 1.4 says that Thompson's group $V$ is of type $F_{\infty}$, which is a well-known result of Brown [6].

Remark 1.5. There is no finite-dimensional topological model for $\mathcal{A}_{q r}^{D}$. If there were such a topological model $X$, then the Higman-Thompson group $F_{q r}$, which is discretely embedded into $\mathcal{A}_{q r}^{D}$ (Remark 4.4), would act properly on a contractible finite-dimensional CW-complex by cell permuting homeomorphisms. Since one can compute the rational homology from $X$ [5, Exercise 2 on p. 174], this would imply $H_{k}\left(F_{q r}, \mathbb{Q}\right)=0$ for all $k$ large enough. But this would contradict the homology computations for $F_{q r}$ in [21, Theorem 4.7]. We refer also to [9, Remark 3.11] and [8, Remark 6.16] for similar statements.

Remark 1.6. Other topological models for Neretin groups which are not of finite type are considered in [13].

Remark 1.7. Our main theorem answers a question of Castellano and Weigel [9, Question 3] in the positive where finiteness conditions are asked for Neretin groups.

One might ask whether $\mathcal{A}_{q r}^{D}$ admits a classifying space of finite type with respect to the family $\mathscr{C} \mathscr{O}$ of compact-open subgroups. Let us first recall this notion.

Definition $1.8([23])$. A (model of the) classifying space of $G$ with respect to a family $\mathcal{F}$ of subgroups of $G$ is a $G$-CW-complex $X$ such that each isotropy group lies in $\mathcal{F}$ and such that for each $H \in \mathcal{F}$ the $H$-fixed point set $X^{H}$ is non-empty and contractible. We denote any such model by $E(G, \mathcal{F})$.

A reductive group $G$ over a local field admits a cocompact model of $E(G, \mathscr{C} \mathscr{O})$; it is given by its Bruhat-Tits building [17, Example 4.14].

Remark 1.9. The group $G=\mathcal{A}_{q r}^{D}$ does not admit a model of $E(G, \mathscr{C} \mathscr{O})$ of finite type. The stabilizer of a point is always subconjugated to the stabilizer of a vertex. Hence, if there was a model with cocompact 0 -skeleton, then every compact-open subgroup of $G$ would be subconjugated to one of the finitely many representatives of conjugacy classes of stabilizers of vertices. By unimodularity there would be a finite upper bound on the Haar measure of compact-open subgroups of $G$. But this cannot be true due to Remark 4.5.

Since a topological model of $G$ that is a $\operatorname{CAT}(0)$-cell complex would be automatically a model of $E(G, \mathscr{C} \mathscr{O})$, any topological model of $G$ with the structure of a CAT(0)-cell complex cannot be cocompact nor of finite type. This was already observed in the paper [8, Remark 6.16] by Caprace and De Medts. 
Remarks 1.5 and 1.9 shows that Theorem 1.4 is the best we can expect in terms of finiteness properties for the groups $\mathcal{A}_{q r}^{D}$.

1.1. Acknowledgments. The initial phase of this project was supported by DFG grant 1661/3-2. Moreover, the second author wants to thank Clara Löh for her hospitality at the University of Regensburg where the first part of this project was carried out. The second author's contribution to this project in the final phase was carried out at the Max Planck Institute for Mathematics in Bonn.

Both authors want to thank Pierre-Emmanuel Caprace, Yves de Cornulier and Adrien Le Boudec for their helpful comments on a draft of this paper.

\section{Notions AND METHODS FROM EQUIVARIANT TOPOLOGY}

2.1. Equivariant $\mathbf{C W}$-complexes. We recall the notion of a $G$-CW-complex as in [23, II.1] or [19, I.1].

Definition 2.1. Let $G$ be a topological group. A $G$ - $C W$-complex is a $G$-space $X$ endowed with a filtration by $G$-subspaces, called skeleta,

$$
X^{(0)} \subset X^{(1)} \subset \ldots \subset X=\bigcup_{n \geq 0} X^{(n)}
$$

such that $X$ carries the colimit topology, the 0-skeleton $X^{(0)}$ is $\bigsqcup_{i \in I_{0}} G / H_{i}$ and for any $n \geq 1$, the $n$-skeleton is obtained from the $(n-1)$-skeleton by a pushout in the category of $G$-spaces

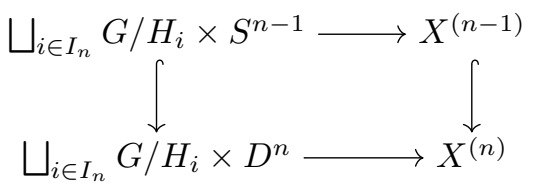

The $H_{i}$ are closed subgroups of $G$. Conjugates of the groups $H_{i}$ are called isotropy groups of $X$. A $G$-CW-complex is called proper or smooth if each $H_{i}$ is compact or open, respectively. It is called finite if $\bigsqcup_{n} I_{n}$ is finite and of finite type if $I_{n}$ is finite for each $n$. The latter is equivalent to the quotient space of every skeleton being compact.

Remark 2.2. A smooth $G$-CW-complex is, forgetting the group action, a CWcomplex. This follows from $G / H$ being discrete whenever $H$ is an open subgroup. Furthermore, the action of $G$ on this CW-complex is continuous and by cell permuting homeomorphisms. An element in $G$ fixing a cell setwise fixes it already pointwise. The stabilizers of cells are the isotropy groups of the $G$-CW-complex.

Vice versa, assume that $X$ is a CW-complex with an action of $G$ with the following properties:

- The action is continuous.

- The action is by cell permuting homeomorphisms.

- An element in $G$ fixing a cell setwise fixes it already pointwise.

- The cell stabilizers are open.

Then $X$ is a smooth $G$-CW-complex with isotropy groups being the cell stabilizers. Hence, if additionally the cell stabilizers are compact, then $X$ is a proper smooth G-CW-complex (cf. [23, Proposition II.1.15]).

Remark 2.3. Let $G$ be a topological group. Let $G^{\delta}$ be $G$ as an abstract group but endowed with the discrete topology. Every smooth $G$-CW-complex $X$ becomes a $G^{\delta}$-CW-complex since $G / H$ is canonically homeomorphic to $G^{\delta} / H$ for every open subgroup $H \leq G$. Vice versa, if $X$ is a $G^{\delta}$-CW-complex such that the isotropy groups are open in $G$, then $X$ is also a smooth $G$-CW-complex. 
Remark 2.4. Because it is a standing assumption in our background reference [19, Convention 1.1 on p. 6] we will assume that topological groups $G$ are compactly generated as spaces in Steenrod's sense 20. This is satisfied if $G$ is a locally compact group. The groups of interest to us, namely tree almost automorphism groups, are locally compact. If we restrict our attention to smooth $G$-CW-complexes, then the discussion in Subsections 2.2 and 2.3 would still be valid for arbitrary topological groups because of Remark 2.3.

Proposition 2.5. Let $X$ be a proper smooth $G$-CW-complex. If $X$ is connected and has a finite 1-skeleton, then $G$ is compactly generated. If, in addition, $X$ is simply connected and has a finite 2-skeleton, then $G$ is compactly presented.

The above proposition is standard in the discrete setting and also not new in the locally compact setting (cf. [10, Theorem 8.A.9]). For convenience of the reader, we point out the short argument based on the well-known lemma below. The lemma does not require that the group $G$ is countable or discrete. Hence we can apply it to a topological group regarded as an abstract group.

Lemma 2.6 (4, Theorem 8.10 on p. 135]). Let $X$ be a topological space, let $G$ be an (abstract) group acting on $X$ by homeomorphisms, and let $U \subset X$ be an open subset such that $G \cdot U=X$.

(1) If $X$ is connected, then $S=\{g \in G \mid g U \cap U \neq \emptyset\}$ generates $G$.

(2) Let $A_{S}$ be a set of symbols $a_{s}$ indexed by $S$. If $X$ and $U$ are both pathconnected and $X$ is simply connected, then $G=\left\langle A_{S} \mid R\right\rangle$ where

$$
R=\left\{a_{s_{1}} a_{s_{2}} a_{s_{3}}^{-1} \mid s_{i} \in S, U \cap s_{1} U \cap s_{3} U \neq \emptyset, s_{1} s_{2}=s_{3} \text { in } G\right\} .
$$

Proof of Proposition 2.5. Let $X$ be a connected proper smooth $G$-CW-complex with finite 1-skeleton $X^{(1)}$. Since $G$ acts cocompactly on $X^{(1)}$ we can find an open, path-connected, relatively compact $U \subset X^{(1)}$ such that $G \cdot U=X^{(1)}$. If, in addition, $X^{(2)}$ is finite, we can find an open, path-connected, relatively compact $U \subset X^{(2)}$ such that $G \cdot U=X^{(2)}$. Since the relations in $R$ are of length 3 , we only have to show that the set $S$ in the statement is relatively compact. But this is clear since the fact that point stabilizers are compact implies that the $G$-action on $X^{(1)}$ resp. $X^{(2)}$ is proper [19, Theorem 1.23 on p. 18].

2.2. Equivariant cell trading. We will introduce an equivariant version of cell trading [11, Proposition 4.2.1]. For this, we will frequently use the following fact:

Remark 2.7. Let $X$ be a space, $Y$ a $G$-space and $H \leq G$ a closed subgroup. Then

$$
\chi: \operatorname{Map}\left(X, Y^{H}\right) \rightarrow \operatorname{Map}^{G}(G / H \times X, Y) \quad f \mapsto[(g H, x) \mapsto g \cdot f(x)]
$$

is a bijection with inverse given by $f \mapsto[x \mapsto f(e H, x)]$.

Now let $(X, A)$ be a pair of $G$-CW-complexes with isotropy groups lying in the family $\mathcal{F}$ which is $(\mathcal{F}, n)$-connected in the following sense.

Definition 2.8. Let $\mathcal{F}$ be a family of subgroups of $G$. We say that a pair $(X, A)$ of $G$-CW-complexes is $(\mathcal{F}, n)$-connected if for each $H \in \mathcal{F}$ the pair $\left(X^{H}, A^{H}\right)$ is $n$-connected. Recall that a pair of spaces $(Y, B)$ is called $n$-connected if each pathconnected component of $Y$ meets $B$ and for each $x_{0} \in B$ and $1 \leq k \leq n$ we have $\pi_{k}\left(Y, B, x_{0}\right)=0$.

Let $e$ be an equivariant $n$-cell in $X$ attached to $A$. We want to explain how we can trade this equivariant cell with an equivariant $(n+2)$-cell of the same isotropy. More precisely, we will construct a pair $\left(X^{t}, A\right)$ of $G$-CW-complexes with isotropy in $\mathcal{F}$ which is $G$-homotopy equivalent to $(X, A)$ and such that $X^{t}$ is obtained from $X$ by deleting $e$ and adding another equivariant $(n+2)$-cell with the same isotropy. 
In the sequel we will freely use the adjunction (2.1). A map to $H$-fixed points will be denoted by lower case letters, and its image under $\chi$ by the corresponding upper case letter.

The equivariant cell $e$ corresponds to a commutative diagram of $G$-maps

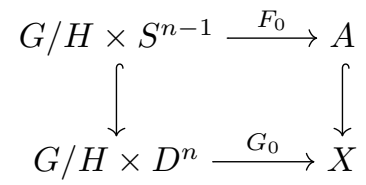

Since the pair $\left(X^{H}, A^{H}\right)$ is assumed to be $n$-connected, there is homotopy of $g_{0}: D^{n} \rightarrow X^{H}$ to a map into $A^{H}$ relative to $f_{0}$. Hence there are maps $g_{1}: D^{n} \rightarrow A^{H}$ and $g_{2}: D^{n+1} \rightarrow X^{H}$ such that $\left.g_{1}\right|_{S^{n-1}}=f_{0}$, such that $g_{2}$ restricted to the upper hemisphere of $\partial D^{n+1}=S^{n}=D^{n} \cup_{S^{n-1}} D^{n}$ is $g_{0}$ and such that $g_{2}$ restricted to the lower hemisphere of $\partial D^{n+1}$ is $g_{1}$. By cellular approximation [3, Lemma 11.2 on p. 207] we may assume that $g_{1}$ lands in the $n$-skeleton and $g_{2}$ lands in the $(n+1)$-skeleton.

For $n \geq 0$ we endow $\left(D^{n+2}, D^{n+1}\right)$ with the following CW-structure: We have one 0-cell lying on the equator $S^{n} \subset D^{n+2}$. The $n$-skeleton is the equator itself, obtained from the 0 -cell by attaching one $n$-cell trivially. Now we attach two $(n+1)$ cells to the $n$-skeleton via the identity $S^{n} \rightarrow S^{n}$ as gluing maps. These two $(n+1)$ cells form the upper and lower hemisphere of $\partial D^{n+2}=S^{n+1}$. We identify the second space in the pair $\left(D^{n+2}, D^{n+1}\right)$ with the lower hemisphere. Finally, we attach one $(n+2)$-cell via the identity $S^{n+1} \rightarrow S^{n+1}$ as gluing map to obtain $D^{n+2}$.

We define $X^{e}$ as the elementary expansion [19, p. 61/62] of $X$ along $G_{2}$, i.e. $X^{e}$ is the following $G$-pushout:

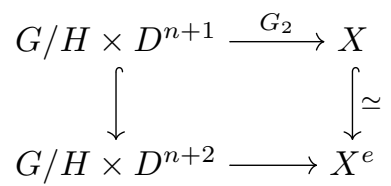

The space $X^{e}$ is obtained from $X$ by attaching an equivariant $(n+1)$-cell corresponding to the upper hemisphere of $S^{n+1}=\partial D^{n+2}$ and an $(n+2)$-cell which defines a $G$-CW-structure on $X^{e}$ (cf. the remarks in [19, p. 62]). The inclusion $X \subset X^{e}$ is a $G$-homotopy equivalence, even a strong $G$-deformation retraction, and the retraction map $p_{X}: X^{e} \rightarrow X$ is cellular [19, p. 62].

Similarly, we define $A^{e}$ as the elementary expansion of $A$ along $G_{1}$ and the corresponding cellular retraction $p_{A}: A^{e} \rightarrow A$ :

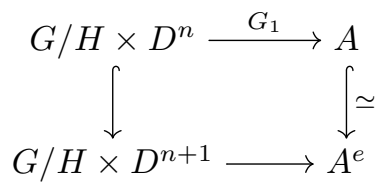

Similarly as above, $A^{e}$ is obtained from $A$ by attaching an equivariant $n$-cell and an equivariant $(n+1)$-cell. The map $p_{A}$ pushes the $n$-cell and the $(n+1)$-cell into $A$. Observe the commutative diagram

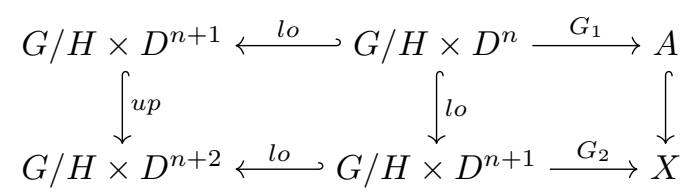


where $u p$ denotes inclusion into the upper hemisphere and $l o$ denotes inclusion into the lower hemisphere. This diagram induces a cellular inclusion $A^{e} \hookrightarrow X^{e}$ of the pushouts of the rows so that

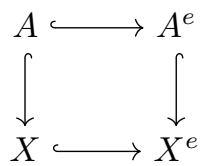

commutes. We define $X^{t}$ by the $G$-pushout:

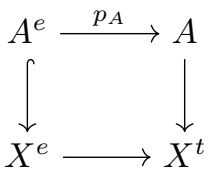

From that pushout, $X^{t}$ inherits the structure of a $G$-CW-complex such that the right vertical map is a cellular inclusion [19, 1.29 on p. 21]. The $n$-cell $e$ gets deleted in $X^{t}$ and so $X^{t}$ has an equivariant $n$-cell less than $X$ but gains an additional equivariant $(n+2)$-cell in exchange. Further, $X^{t}$ and $X$ have the same number of equivariant $k$-cells for $n \neq k \neq n+2$.

Since $A^{e} \hookrightarrow X^{e}$ is a $G$-cofibration [19, 1.5 on p. 8] and $p_{A}$ is a $G$-homotopy equivalence, also $X^{e} \rightarrow X^{t}$ is a $G$-homotopy equivalence. This is a special case of [19, Lemma 2.13 on p. 38] where one takes as lower row $X^{e} \leftarrow A^{e} \stackrel{p_{A}}{\longrightarrow} A^{e}$ whose pushout is $X^{t}$ and as upper row $X^{e} \leftarrow A^{e} \stackrel{\text { id }}{\rightarrow} A^{e}$ whose pushout is $X^{e}$; the maps from the upper to the lower row are $\mathrm{id}_{X^{e}}, \mathrm{id}_{A^{e}}$, and $p_{A}$. By putting the squares in (2.2) and (2.3) next to each other we obtain a bigger commutative diagram whose bottom row yields a $G$-homotopy equivalence $X \rightarrow X^{t}$ which is compatible with the two embeddings of $A$, i.e. we have a commutative triangle:

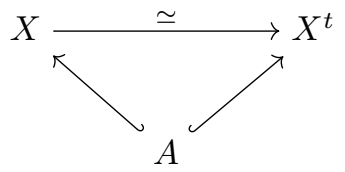

We say that $X^{t}$ results from trading the equivariant cell e in $X$ (relative to $A$ ).

2.3. Cell trading on a filtration. Let $X$ be a $G$-CW-complex with isotropy lying in the family $\mathcal{F}$. Consider a filtration $X_{0} \subset X_{1} \subset \ldots \subset X$ of $X$ by $G$-subcomplexes with $\bigcup_{i>0} X_{i}=X$. We define this filtration to be $\mathcal{F}$-highly connected if the $\mathcal{F}$ connectivity of the pairs $\left(X_{i+1}, X_{i}\right)$ tends to infinity as $i \rightarrow \infty$, i.e.

$$
\forall_{k} \exists_{n} \forall_{i \geq n}\left(X_{i+1}, X_{i}\right) \text { is }(\mathcal{F}, k) \text {-connected. }
$$

We say that this filtration is of finite type if each $X_{i}$ is of finite type in the equivariant sense.

Theorem 2.9. Let $X$ be a $G$-CW-complex with isotropy in $\mathcal{F}$ and with a $\mathcal{F}$-highly connected finite type filtration $\left(X_{i}\right)_{i \geq 0}$. Then $X$ is $G$-homotopy equivalent to a finite type $G$-CW-complex with isotropy in $\mathcal{F}$.

Proof. First we find a sequence of numbers $n_{0}<n_{1}<n_{2}<\ldots$ such that $\left(X_{i+1}, X_{i}\right)$ is $(\mathcal{F}, k)$-connected for each $i \geq n_{k}$. Upon passage to the sparser filtration $X_{n_{1}} \subset$ $X_{n_{2}} \subset \ldots$ and renumbering we may assume that $n_{k}=k$. In the following we denote the $i$-skeleton of a $G$-CW-complex $Y$ by $\operatorname{sk}_{i}(Y)$. 
We inductively build the following commutative diagram of $G$-CW-complexes of finite type and with isotropy in $\mathcal{F}$ :

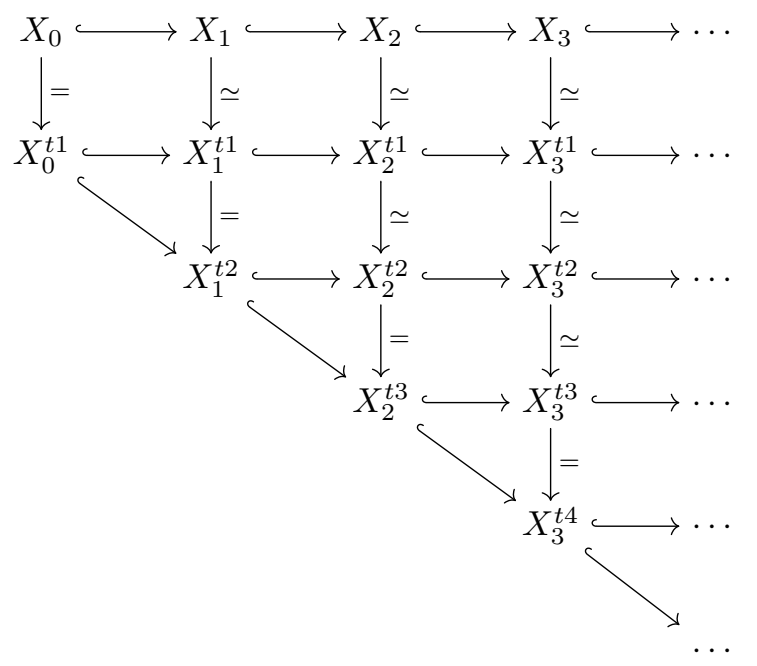

We enumerate the rows starting with 0 . Further properties are:

(1) Arrows labeled with $\simeq$ are $G$-homotopy equivalences and those with a hook indicate cellular inclusions.

(2) For $l \geq 1$ the $(l-1)$-skeleton of each space in the row $l$ is $\operatorname{sk}_{l-1}\left(X_{l-1}^{t l}\right)$.

Next we explain how to construct the $l$-th row from the $(l-1)$-th row. For a uniform treatment of the start case and the induction step, set $X_{i}^{t 0}:=X_{i}$, and let $l \geq 1$. For $i \geq l-1$ the pair $\left(X_{i}^{t(l-1)}, X_{l-1}^{t(l-1)}\right)$ is $(\mathcal{F}, l-1)$-connected. The boundary of an $(l-1)$-cell in $X_{i}^{t(l-1)} \backslash X_{l-1}^{t(l-1)}, l \geq 2$, lies in

$$
\operatorname{sk}_{l-2}\left(X_{i}^{t(l-1)}\right)=\operatorname{sk}_{l-2}\left(X_{l-2}^{t(l-1)}\right) \subset X_{l-1}^{t(l-1)} .
$$

Hence we may and will trade every equivariant $(l-1)$-cell in $X_{i}^{t(l-1)} \backslash X_{l-1}^{t(l-1)}$ with an equivariant $(l+1)$-cell resulting in a $G$-CW-complex $X_{i}^{t l}$ of finite type and isotropy in $\mathcal{F}$ and a $G$-homotopy equivalence $X_{i}^{t(l-1)} \rightarrow X_{i}^{t l}$. Doing so successively, i.e. first trading all $(l-1)$-cells lying in $X_{l}^{t(l-1)} \backslash X_{l-1}^{t(l-1)}$ in the complexes $X_{l}^{t(l-1)}, X_{l+1}^{t(l-1)}, \ldots$, then trading all $(l-1)$-cells lying in $X_{l+1}^{t(l-1)} \backslash X_{l}^{t(l-1)}$ in the complexes $X_{l+1}^{t(l-1)}, X_{l+2}^{t(l-1)}, \ldots$ etc., results in a commutative ladder which gives the $l$-th row:

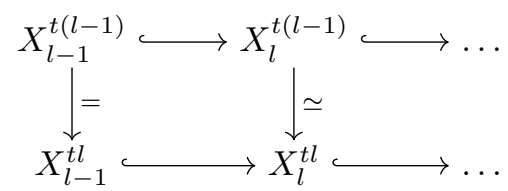

It is clear from the construction that the $(l-1)$-skeleton of $X_{i}^{t l}$ coincides with the one of $X_{l-1}^{t l}$. The colimit $Y$ of the diagonal arrows in (2.4) is a $G$-CW complex of finite type whose isotropy groups are in $\mathcal{F}$. Finally, we obtain a commutative diagram

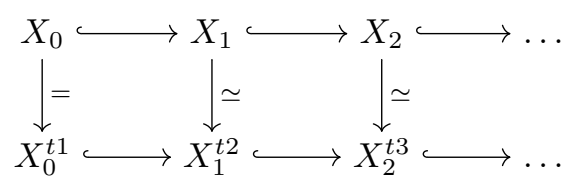


where the vertical arrows are the compositions of the corresponding column arrows in (2.4). Since the horizontal arrows are $G$-cofibrations and each vertical arrow is a $G$-homotopy equivalence, the induced map between the colimits of the rows, i.e. between $X$ and $Y$, is a $G$-homotopy equivalence [24, Theorem 1.2].

\section{Generalized posets and Morse theory}

In the following, we will always implicitly regard categories as topological objects via the nerve construction. More precisely, the nerve of a category is a simplicial set and the geometric realization of this simplicial set is a CW-complex built up from simplices glued along their faces (it is, however, not always a simplicial complex). The 0-simplices of this complex are the objects of the category and the $n$-cells are in one to one correspondence with sequences of $n$ composable non-identity arrows

$$
A_{0} \stackrel{\alpha_{1}}{\longrightarrow} A_{1} \stackrel{\alpha_{2}}{\longrightarrow} \cdots \stackrel{\alpha_{n}}{\longrightarrow} A_{n}
$$

The top-dimensional faces of such a simplex are the simplices

$$
\begin{gathered}
A_{1} \stackrel{\alpha_{2}}{\longrightarrow} A_{2} \stackrel{\alpha_{3}}{\longrightarrow} \cdots \stackrel{\alpha_{n}}{\longrightarrow} A_{n} \\
A_{0} \stackrel{\alpha_{1}}{\longrightarrow} \cdots A_{k-1} \stackrel{\alpha_{k+1} \circ \alpha_{k}}{\longrightarrow} A_{k+1} \cdots \stackrel{\alpha_{n}}{\longrightarrow} A_{n} \\
A_{0} \stackrel{\alpha_{1}}{\longrightarrow} \cdots \stackrel{\alpha_{n-2}}{\longrightarrow} A_{n-2} \stackrel{\alpha_{n-1}}{\longrightarrow} A_{n-1}
\end{gathered}
$$

for $k=1, \ldots, n-1$. For the faces of the second type, it is possible that the composition $\alpha_{k+1} \circ \alpha_{k}$ is the identity. In this case, the identity arrow is deleted in the sequence and we obtain a degeneracy in the gluing of the $n$-simplex.

For example, a partially ordered set (poset) can be regarded as a category $\mathcal{C}$ with

$$
\left|\operatorname{Hom}_{\mathcal{C}}(A, B) \cup \operatorname{Hom}_{\mathcal{C}}(B, A)\right| \leq 1
$$

for every two objects $A, B$. In this case, the geometric realization of $\mathcal{C}$ is indeed a simplicial complex. However, we also want to work with complexes which arise from a slightly bigger class of categories:

Definition 3.1. A generalized poset is a small category such that $\alpha=\beta$ whenever $\alpha, \beta: A \rightarrow B$.

Hence in a generalized poset, we allow objects to be uniquely isomorphic. Every subgroupoid of a generalized poset has trivial automorphism groups and is therefore equivalent to a disjoint union of terminal categories. Thus, every connected component of such a subgroupoid is contractible (recall that we implicitly regard categories as topological objects). Since the inclusion of subcategories are inclusions of subcomplexes on the level of spaces and hence cofibrations, we can collapse connected subgroupoids in generalized posets and obtain homotopy equivalent generalized posets. If we collapse each connected component of the full subgroupoid consisting of all the isomorphisms, we even get a homotopy equivalent (honest) poset which we call the underlying poset of the generalized poset.

More precisely, let $\mathcal{C}$ be a generalized poset and $\mathcal{G}$ a subgroupoid. We define the category $\mathcal{C} / \mathcal{G}$ as follows: The objects of $\mathcal{C} / \mathcal{G}$ are equivalence classes of objects of $\mathcal{C}$ where we say that $X \sim Y$ are equivalent if there is an isomorphism $X \rightarrow Y$ in $\mathcal{G}$ (which is unique). We define

$$
\operatorname{Hom}_{\mathcal{C} / \mathcal{G}}([X],[Y]):=\{A \rightarrow B \text { in } \mathcal{C} \mid A \in[X], B \in[Y]\} / \sim
$$


where elements $(A \rightarrow B) \sim\left(A^{\prime} \rightarrow B^{\prime}\right)$ are defined to be equivalent if the diagram

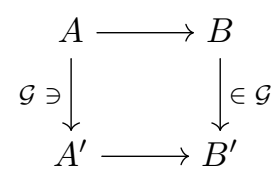

commutes. Let $[\alpha: A \rightarrow B]$ and $[\beta: C \rightarrow D]$ be two composable arrows, i.e. $[B]=$ $[C]$, then there is a unique isomorphism $\gamma: B \rightarrow C$ in $\mathcal{G}$ and one defines

$$
[\beta: C \rightarrow D] \circ[\alpha: A \rightarrow B]:=[\beta \circ \gamma \circ \alpha: A \rightarrow D] .
$$

Set $\operatorname{id}_{[X]}=\left[\operatorname{id}_{X}\right]$. The next proposition is probably well-known and can be quickly deduced from Quillen's Theorem A (see [22, Proposition 2.8] for a full proof).

Proposition 3.2. The projection $\mathcal{C} \rightarrow \mathcal{C} / \mathcal{G}$ is a functor which induces a homotopy equivalence on the level of spaces.

We recall some more category-theoretic notions. The (biased) join $\mathcal{C} * \mathcal{D}$ of two categories is the unique category containing $\mathcal{C}$ and $\mathcal{D}$ as subcategories and exactly one extra arrow $C \rightarrow D$ for each two objects $C \in \mathcal{C}$ and $D \in \mathcal{D}$. Further, Coone $(\mathcal{C} * \mathcal{D})$ is the unique category containing $\mathcal{C} * \mathcal{D}$ as a subcategory, containing an extra object named tip, an extra arrow $C \rightarrow$ tip for each object $C \in \mathcal{C}$ and an extra arrow tip $\rightarrow D$ for each object $D \in \mathcal{D}$. On the level of spaces, $\mathcal{C} * \mathcal{D}$ is homotopy equivalent to the join of (the nerves of) $\mathcal{C}$ and $\mathcal{D}$, and $\operatorname{Coone}(\mathcal{C} * \mathcal{D})$ is homotopy equivalent to the cone over (the nerve of) $\mathcal{C} * \mathcal{D}$, thus contractible.

If $\mathcal{C}$ is a category and $X$ an object in $\mathcal{C}$, then $X \downarrow \mathcal{C}$ is the category whose objects are $\mathcal{C}$-morphisms $X \rightarrow Y$ and whose morphisms are $\mathcal{C}$-morphisms below $X$. Similary, one defines $\mathcal{C} \downarrow X$ as the category whose objects are $\mathcal{C}$-morphisms $Y \rightarrow X$ and whose morphisms are $\mathcal{C}$-morphisms over $X$.

Remark 3.3. Let $G$ be a group acting on a small category $\mathcal{C}$ by invertible functors. An element $g \in G$ fixing a cell setwise already fixes its vertices and so fixes the cell pointwise. If $\mathcal{C}$ is a generalized poset, a cell stabilizer is equal to the intersection of the vertex stabilizers of that cell.

Remark 3.4. Let $G$ act on a generalized poset $\mathcal{C}$. Then denote by $\mathcal{C}^{G}$ the full subcategory spanned by the objects fixed by $G$. The nerve of $\mathcal{C}^{G}$ is the subspace of $G$-fixed points in the nerve of $\mathcal{C}$. Hence the generalized poset $\mathcal{C}^{G}$ is the correct category representing the $G$-fixed point set in the nerve of $\mathcal{C}$.

Now we review the discrete Morse method for categories from 22, Subsection $2.10]$ in the case of generalized posets. Let $\mathcal{C}$ be a generalized poset and $\mathcal{A} \subset \mathcal{C}$ a full subcategory. We are inductively adding objects to $\mathcal{A}$ to build up $\mathcal{C}$. We start with $\mathcal{C}_{0}:=\mathcal{A}$. Assume we have already constructed $\mathcal{C}_{n}$. Let $X$ be an object in $\mathcal{C} \backslash \mathcal{C}_{n}$ and observe the full subcategory $\mathcal{C}_{n+1}$ spanned by $\mathcal{C}_{n}$ and the object $X$.

Case 1: Assume that there is an isomorphism connecting $X$ with an element in $\mathcal{C}_{n}$. Then the inclusion $\mathcal{C}_{n} \rightarrow \mathcal{C}_{n+1}$ is a homotopy equivalence by [22, Lemma 2.18].

Case 2: Assume that there is no isomorphism connecting $X$ with an element in $\mathcal{C}_{n}$. Then for every object $Y \in \mathcal{C}_{n}$ there are either no arrows or only one arrow connecting $X$ with $Y$. By [22, Lemma 2.19] the diagram

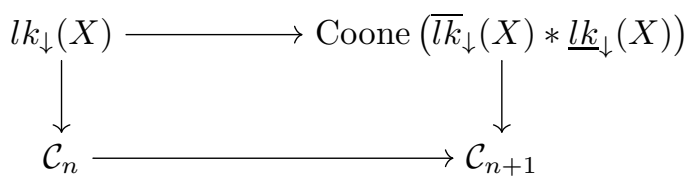


is a pushout on the level of spaces (in general not a pushout in the category of small categories). Here, $l k_{\downarrow}(X):=\overline{l k}_{\downarrow}(X) * \underline{l k}_{\downarrow}(X)$ is the descending link of $X$ with $\overline{l k}_{\downarrow}(X):=\mathcal{C}_{n} \downarrow X$ and $\underline{l k_{\downarrow}}(X):=X \downarrow \mathcal{C}_{n}$. The above pushout implies:

Remark 3.5. In (3.1) the upper horizontal map is a cofibration since it is an inclusion of subcomplexes. Hence the Blakers-Massey homotopy excision theorem implies that the pair $\left(\mathcal{C}_{n+1}, \mathcal{C}_{n}\right)$ is $(k+1)$-connected provided the descending link $l k_{\downarrow}(X)$ is $k$-connected.

If we have enough control over the connectivity of the descending links appearing in the process, we can ultimately deduce a lower connectivity bound for the pair $(\mathcal{C}, \mathcal{A})$. The obtained bound crucially depends on the order in which the objects are added. Such an order is often encoded in a so-called Morse function:

Definition 3.6. A generalized Morse function on the pair $(\mathcal{C}, \mathcal{A})$ is a function $f$ assigning a natural number to objects in $\mathcal{C} \backslash \mathcal{A}$ such that no two objects with the same $f$-value are connected by a non-invertible arrow. We call such $f$ well-behaved if no two objects of different Morse height are joined by an isomorphism.

We then add objects by increasing $f$-value. Note that two objects $X_{1}, X_{2}$ may have the same $f$-value. We claim that the descending links do not depend on the adding order of such objects. This is easy to see if $X_{1}, X_{2}$ are not joined by any arrow. If there are (unique) isomorphisms between $X_{1}$ and $X_{2}$, then the descending link $l k_{\downarrow}\left(X_{1}\right)$ (when adding $X_{1}$ first) is isomorphic to the descending link $l k_{\downarrow}\left(X_{2}\right)$ (when adding $X_{2}$ first) via the unique isomorphisms between $X_{1}$ and $X_{2}$.

So we see that when adding objects of a new Morse level, we have to check one descending link for each isomorphism component of the new level. More precisely, we first add all the objects which are joined by isomorphisms to the lower level. This gives homotopy equivalences by the first case above. Then we add an object which is not joined by an isomorphism to the lower level and have to check the corresponding descending link. We then add all the objects joined by an isomorphism to this object which again gives homotopy equivalences. We continue with an object of another isomorphism component and repeat this process.

Remark 3.7. A well-behaved generalized Morse function $f$ on $\mathcal{C}$ descends to a (honest) Morse function $f^{\prime}$ (i.e. no two objects of the same height are joined by an arrow) on the underlying poset $\mathcal{C}^{\prime}$ of $\mathcal{C}$. The underlying posets of the descending links with respect to $f$ are the descending links with respect to $f^{\prime}$ (more precisely, this is true for the descending link of each vertex of an isomorphism component in $\mathcal{C}$ which is added first). By Proposition [3.2, checking the connectivity of the descending links in a generalized poset is the same as checking the connectivity of the descending links in its underlying poset.

\section{Tree Almost AUtOMORPhism Groups}

4.1. Review of tree almost automorphism groups. For $q \geq 2$, we denote by $T_{q}$ the rooted $q$-regular tree, i.e. the tree with root $o$ of degree $q$ and other vertices with degree $q+1$. We fix an embedding of $T_{q}$ in the plane. The embedding induces a canonical ordering, say from left to right, of the direct descendants of any vertex. The induced lexicographic order is a total order on the space of ends $B_{q}=\partial T_{q}$. We endow $B_{q}$ with the visual metric $d$ defined by

$$
d(\xi, \eta)=\exp \left(-(\xi, \eta)_{o}\right)
$$

where $(\xi, \eta)_{o}$ is the length of the common initial segment of the infinite paths that start at the root $o$ and represent $\xi$ and $\eta$. A ball in $B_{q}$ is represented by a canonical 
subtree of $T_{q}$ containing some vertex and all of its descendants. Such a subtree is again a rooted $q$-regular tree.

Let $\operatorname{Aut}\left(T_{q}\right)$ be the group of isometries of $T_{q}$. We endow $\operatorname{Aut}\left(T_{q}\right)$ with the compact open topology. A neighborhood basis of id $\in \operatorname{Aut}\left(T_{q}\right)$ is given by the subsets

$$
U_{n}=\left\{\gamma \in \operatorname{Aut}\left(T_{q}\right)|\gamma|_{B(o, n)}=\operatorname{id}_{B(o, n)}\right\}
$$

With this topology, $\operatorname{Aut}\left(T_{q}\right)$ is a topological group which is totally disconnected and, by the Theorem of Arzelà-Ascoli, compact. It is isomorphic, as a topological group, to the group of isometries of the space of ends $B_{q}$ endowed with the compact open topology, i.e. $\operatorname{Aut}\left(T_{q}\right)=\operatorname{Isom}\left(B_{q}\right)$ for short.

A similarity $\phi: X \rightarrow Y$ of metric spaces is a homeomorphism $\phi$ such that there is a $\lambda>0$ with $d\left(\phi\left(x_{1}\right), \phi\left(x_{2}\right)\right)=\lambda d\left(x_{1}, x_{2}\right)$ for all $x_{1}, x_{2} \in X$. We call it a local similarity if it is a homeomorphism and if for each $x \in X$ there is $r>0$ and $\lambda>0$ such that

$$
\left.\phi\right|_{B(x, r)}: B(x, r) \rightarrow B(f(x), \lambda r)
$$

is a $\lambda$-similarity. We call $\lambda$ the slope of $\phi$ at $x$. In the case of our rooted $q$-regular tree $T_{q}$ with space of ends $B_{q}$, we call a local similarity of $B_{q}$ a spheromorphism. If $\phi: B_{q} \rightarrow B_{q}$ is such a spheromorphism, we always find finite rooted $q$-regular subtrees $F_{1}, F_{2} \subset T_{q}$ and an isometry of forests $f: T_{q} \backslash F_{1} \rightarrow T_{q} \backslash F_{2}$ which induces $\phi$. We say that $f$ represents $\phi$. Consult 12 for a detailed exposition of such correspondences.

Definition 4.1. We define $\mathcal{A}_{q}$ to be the subgroup of $\operatorname{Homeo}\left(B_{q}\right)$ consisting of all spheromorphisms. We endow it with the unique group topology such that the inclusion $\operatorname{Isom}\left(B_{q}\right) \hookrightarrow \mathcal{A}_{q}$ is continuous and open.

With this topology, $\mathcal{A}_{q}$ is totally disconnected and locally compact. It is simply the topology where the sets $U_{n} \subset \operatorname{Isom}\left(B_{q}\right) \subset \mathcal{A}_{q}$ still form a neighborhood basis of id $\in \mathcal{A}_{q}$. A neighborhood basis of $\gamma \in \mathcal{A}_{q}$ is thus formed by the sets $\gamma U_{n}$. To see that multiplication is continuous, let $\gamma_{1}, \gamma_{2} \in \mathcal{A}_{q}$ and $n \in \mathbb{N}$. We can choose $n^{\prime} \in \mathbb{N}$ such that $U_{n^{\prime}} \gamma_{2} \subset \gamma_{2} U_{n}$ (Remark 4.7). Then we have

$$
\gamma_{1} U_{n^{\prime}} \gamma_{2} U_{n} \subset \gamma_{1} \gamma_{2} U_{n} U_{n}=\gamma_{1} \gamma_{2} U_{n}
$$

For the inverses, let $\gamma \in \mathcal{A}_{q}$ and $n \in \mathbb{N}$. We can choose $n^{\prime} \in \mathbb{N}$ such that $\gamma U_{n^{\prime}} \subset U_{n} \gamma$ (Remark 4.7). Then we have

$$
\left(\gamma U_{n^{\prime}}\right)^{-1} \subset\left(U_{n} \gamma\right)^{-1}=\gamma^{-1} U_{n}^{-1}=\gamma^{-1} U_{n}
$$

We now want to generalize these groups in two steps. For the first step, we need the following terminology: For two metric spaces $X$ and $Y$, let $X \sqcup Y$ be the disjoint union of $X$ and $Y$ with the unique metric $d$ extending the metrics on $X$ and $Y$ and with $d(x, y)=\infty$ for $x \in X$ and $y \in Y$. By $n X$ we denote the disjoint union $n X=X \sqcup \ldots \sqcup X$ of $n$ copies of $X$. Note that the summands in such a disjoint union are ordered. Thus, if in addition $X$ is ordered (for example if $X=B_{q}$ is the space of ends of $T_{q}$ from above), we get an ordering on $n X$.

Let $r \geq 1$. Generalizing the notion of spheromorphisms, we call a local similarity $r B_{q} \rightarrow r B_{q}$ an $r$-spheromorphism. Observe that the elements in $\operatorname{Isom}\left(B_{q}\right)^{r}$ are $r$-spheromorphisms in a canonical way. We endow $\operatorname{Isom}\left(B_{q}\right)^{r}$ with the product topology. Then the sets $\left(U_{n}\right)^{r} \subset \operatorname{Isom}\left(B_{q}\right)^{r}$ for varying $n$ form a neighborhood basis of id $\in \operatorname{Isom}\left(B_{q}\right)^{r}$.

Definition 4.2. We define $\mathcal{A}_{q r}$ to be the subgroup of $\operatorname{Homeo}\left(r B_{q}\right)$ consisting of all $r$-spheromorphisms. We endow it with the unique group topology such that the inclusion $\operatorname{Isom}\left(B_{q}\right)^{r} \hookrightarrow \mathcal{A}_{q r}$ is continuous and open. 
With this topology, $\mathcal{A}_{q r}$ is totally disconnected and locally compact. A neighborhood basis of $\gamma \in \mathcal{A}_{q r}$ is formed by the sets $\gamma\left(U_{n}\right)^{r}$. Obviously, we have $\mathcal{A}_{q}=\mathcal{A}_{q 1}$.

For the second step, we look at certain closed subgroups of $\operatorname{Isom}\left(B_{q}\right)$ : Let $D \leq \operatorname{Sym}(q)$ be a subgroup of the group of permutations on the set with $q$ elements. Observe that an element $\gamma \in \operatorname{Aut}\left(T_{q}\right)$ induces a permutation of the $q$ direct descendants of any vertex $v \in T_{q}$ (after canonically identifying the full subtree below the vertex $v$ with the full subtree below the vertex $\gamma(v)$ via the unique order-preserving tree automorphism mapping $v$ to $\gamma(v))$. We say that $\gamma \in \operatorname{Aut}\left(T_{q}\right)$ is $D$-admissible if all these permutations are elements in $D$. The set of all $D$-admissible elements in $\operatorname{Aut}\left(T_{q}\right)=\operatorname{Isom}\left(B_{q}\right)$ forms a closed subgroup of $\operatorname{Isom}\left(B_{q}\right)$ which we denote by $\operatorname{Isom}_{D}\left(B_{q}\right)$ and which is denoted by $W(D)$ in [8] and [16]. Hence also $\operatorname{Isom}_{D}\left(B_{q}\right)$ is totally disconnected and compact. A neighborhood basis of the identity is formed by the sets $U_{n}^{D}:=\operatorname{Isom}_{D}\left(B_{q}\right) \cap U_{n}$.

Let $U, V \subset B_{q}$ be balls and $\phi: U \rightarrow V$ a similarity. Since $U, V$ are represented by canonical subtrees and $\phi$ by an isometry of these trees, it is clear what it means for $\phi$ to be $D$-admissible. Consequently, we can define an $r$-spheromorphism to be $D$-admissible if it is locally determined by $D$-admissible similarities $\phi: U \rightarrow V$ where $U, V$ are balls in $r B_{q}$.

Definition 4.3. We define $\mathcal{A}_{q r}^{D}$ to be the subgroup of Homeo $\left(r B_{q}\right)$ consisting of all $r$-spheromorphisms which are $D$-admissible. We endow it with the unique group topology such that the inclusion $\operatorname{Isom}_{D}\left(B_{q}\right)^{r} \hookrightarrow \mathcal{A}_{q r}^{D}$ is continuous and open.

With this topology, $\mathcal{A}_{q r}^{D}$ is totally disconnected and locally compact. A neighborhood basis of $\gamma \in \mathcal{A}_{q r}^{D}$ is formed by the sets $\gamma\left(U_{n}^{D}\right)^{r}$. Obviously, we have $\mathcal{A}_{q r}=\mathcal{A}_{q r}^{\operatorname{Sym}(q)}$. Moreover, $\mathcal{A}_{q r}^{\{1\}}$ is the discrete Higman-Thompson group $V_{q r}$.

Remark 4.4. The Higman-Thompson groups $F_{q r}$ and $V_{q r}$ canonically embed into $\mathcal{A}_{q r}^{D}$ for any $D \leq \operatorname{Sym}(q)$. In the first case the embedding is discrete and dense in the second. For $D=\{1\}$, this is trivial. For $D \neq\{1\}$, it suffices to check the following:

- $\operatorname{Isom}_{D}\left(B_{q}\right)^{r} \cap F_{q r}=\left\{\operatorname{id}_{r B}\right\}$

- $\forall_{n \in \mathbb{N}}: U_{n}^{D} \cap V_{q r} \supsetneq\left\{\operatorname{id}_{r B}\right\}$

Remark 4.5. Let $O_{n} \subset \mathcal{A}_{q r}^{D}$ be the subgroup of $D$-admissible $r$-spheromorphisms which are represented by an isometry of the forest $T_{q} \backslash B(o, n) \sqcup \ldots \sqcup T_{q} \backslash B(o, n)$. The subgroup $O_{n}$ is open and compact, and the increasing union $O=\bigcup O_{n}$ is non-compact. In particular, the Haar measures of the $O_{n}$ tend to $\infty$.

In the following discussion we will fix $q \geq 2, r \geq 1$ and $D \leq \operatorname{Sym}(q)$. For better readability, we will often drop these symbols from the notation. For example, we will write $\mathcal{A}, T, B$ instead of $\mathcal{A}_{q r}^{D}, T_{q}, B_{q}$.

4.2. Generalized posets for tree almost automorphism groups. We will now define a generalized poset $\mathcal{R}$ for the group $\mathcal{A}$ and consider two quotients $\mathcal{Q}$ and $\mathcal{P}$.

\section{Definition 4.6.}

- A sphero-vertex is a local similarity $\phi: m B \rightarrow n B$ for $m, n \geq 1$ which is $D$-admissible, i.e. which is locally determined by $D$-admissible similarities of subballs. We say that $m=\operatorname{lvl}(\phi)$ is the level of $\phi$.

- For $m>n$, a merge map $\mu: m B \rightarrow n B$ is a $D$-admissible local similarity such that for each summand $B$ in the domain $m B$ the restriction $\left.\mu\right|_{B}: B \rightarrow$ $C$ is a $D$-admissible similarity onto a ball $C$ in the codomain $n B$.

- We say that such a merge map is very elementary if for each summand $B$ in the codomain $n B$, the preimage $\mu^{-1}(B)$ consists of at most $q$ summands in the domain $m B$ (in other words, it is exactly one or exactly $q$ summands). 
- A (very elementary) split map is the inverse of a (very elementary) merge map.

- A transformation is a $D$-admissible isometry $\nu: n B \rightarrow n B$ with $n \geq 1$. In other words, there is a permutation $\sigma \in \operatorname{Sym}(n)$ such that $\nu$ maps the $i$ 'th summand in the domain $n B$ onto the $\sigma(i)$ 'th summand in the codomain $n B$ via an element in $\operatorname{Isom}_{D}(B)$.

- A strict transformation is a D-admissible isometry $\nu: n B \rightarrow n B$ with $\sigma=$ id. In other words, a strict transformation is simply an element in $\operatorname{Isom}_{D}(B)^{n}$.

Remark 4.7. An important feature of tree almost automorphism groups is a certain subnormality condition: Whenever $k \in \mathbb{N}$ and $\phi: m B \rightarrow n B$ is a sphero-vertex, then we find $k^{\prime} \in \mathbb{N}$ big enough so that $\phi \circ\left(U_{k^{\prime}}^{D}\right)^{m} \circ \phi^{-1} \subset\left(U_{k}^{D}\right)^{n}$. We have already used this to prove that multiplication and taking inverses in $\mathcal{A}$ is continuous.

The elements of the generalized poset $\mathcal{R}$ are the sphero-vertices $n B \rightarrow r B$ for varying $n \geq 1$ (recall that $r \geq 1$ is fixed). An arrow from a sphero-vertex $\phi: n B \rightarrow$ $r B$ to a sphero-vertex $\psi: m B \rightarrow r B$ with $n \geq m$ is either a merge map or a transformation $\alpha$ with $\psi \circ \alpha=\phi$. Composition is given by composing the merge maps resp. transformations. Note that the composition of a merge map with a transformation is again a merge map. The identities in $\mathcal{R}$ are given by the identity transformations.

Let $\mathcal{T}$ be the subgroupoid of $\mathcal{R}$ containing all the isomorphisms, i.e. all the transformations. Then $\mathcal{P}:=\mathcal{R} / \mathcal{T}$ is the underlying poset of $\mathcal{R}$. Let $\mathcal{S}$ be the subgroupoid of $\mathcal{R}$ containing all the strict transformations. Then $\mathcal{Q}:=\mathcal{R} / \mathcal{S}$ is a generalized poset.

The level function is a well-behaved generalized Morse function on $\mathcal{R}$ and so descends to a generalized Morse function on $\mathcal{Q}$ and to a Morse function on $\mathcal{P}$. Let $\mathcal{R}(n)$ resp. $\mathcal{Q}(n)$ resp. $\mathcal{P}(n)$ be the full subposets spanned by the objects of level at most $n$.

We define an action of $\mathcal{A}$ on $\mathcal{R}$ from the left by $\gamma \cdot \phi:=\gamma \circ \phi$ where $\gamma \in \mathcal{A}$ and $\phi$ is a sphero-vertex. Observe that this is a free action. It induces an action on $\mathcal{Q}$ resp. $\mathcal{P}$ via $\gamma \cdot[\phi]:=[\gamma \circ \phi]$. Since this action preserves the level of elements, the Morse filtrations $\mathcal{R}(n), \mathcal{Q}(n)$ and $\mathcal{P}(n)$ are $\mathcal{A}$-invariant.

Remark 4.8. Let $[\phi]$ be an object in $\mathcal{Q}$ or $\mathcal{P}$ with $\phi: n B \rightarrow r B$ a sphero-vertex. By Remark 4.7, we can find $k \in \mathbb{N}$ big enough such that $\left(U_{k}^{D}\right)^{r} \circ \phi \subset \phi \circ \operatorname{Isom}_{D}(B)^{n}$ and hence $\gamma \cdot[\phi]=[\phi]$ for each $\gamma \in\left(U_{k}^{D}\right)^{r}$. This shows that the action of $\mathcal{A}$ on $\mathcal{Q}$ and on $\mathcal{P}$ is continuous (compare also with Remark 3.3. $)$.

\subsection{Properties of the generalized posets.}

Proposition 4.9. The cell stabilizers of the action of $\mathcal{A}$ on $\mathcal{Q}$ are compact and open.

Proof. Since the stabilizer of a cell is the intersection if its vertex stabilizers (Remark 3.3), it is sufficient to prove the statement for the vertex stabilizers. So let $[\phi]$ be an object in $\mathcal{Q}$ and $\gamma \in \mathcal{A}$ with $\gamma \cdot[\phi]=[\phi]$. We obtain a strict transformation $\nu: n B \rightarrow n B$ with $\gamma \circ \phi=\phi \circ \nu$, i.e. $\gamma=\phi \circ \nu \circ \phi^{-1}$. Conversely, it is easy to see that an element of this form stabilizes $[\phi]$. Hence we have

$$
\operatorname{Stab}[\phi]=\left\{\phi \circ \nu \circ \phi^{-1} \mid \nu \text { strict transformation }\right\}
$$

The group of strict transformations of $n B$ is isomorphic to $\operatorname{Isom}_{D}(B)^{n}$ which we endow with the product topology. The image of the injective group homomorphism

$$
\iota: \operatorname{Isom}_{D}(B)^{n} \rightarrow \mathcal{A} \quad \nu \mapsto \phi \circ \nu \circ \phi^{-1}
$$


is equal to $\operatorname{Stab}[\phi]$ and it suffices to show that $\iota$ is continuous and open.

Concerning continuity, let $k \in \mathbb{N}$. By Remark 4.7] we find $k^{\prime} \in \mathbb{N}$ big enough so that

$$
\left(U_{k^{\prime}}^{D}\right)^{n} \circ \phi^{-1} \subset \phi^{-1} \circ\left(U_{k}^{D}\right)^{r}
$$

For $\gamma_{1}, \ldots, \gamma_{n} \in \operatorname{Isom}_{D}(B)$ and $\alpha_{1}, \ldots, \alpha_{n} \in U_{k^{\prime}}^{D}$ we then have

$$
\begin{aligned}
\iota\left(\gamma_{1} \alpha_{1}, \ldots, \gamma_{n} \alpha_{n}\right) & =\phi\left(\gamma_{1} \alpha_{1}, \ldots, \gamma_{n} \alpha_{n}\right) \phi^{-1} \\
& =\phi\left(\gamma_{1}, \ldots, \gamma_{n}\right)\left(\alpha_{1}, \ldots, \alpha_{n}\right) \phi^{-1} \\
& =\phi\left(\gamma_{1}, \ldots, \gamma_{n}\right) \phi^{-1}\left(\beta_{1}, \ldots, \beta_{r}\right) \\
& =\iota\left(\gamma_{1}, \ldots, \gamma_{n}\right)\left(\beta_{1}, \ldots, \beta_{r}\right)
\end{aligned}
$$

for suitable $\beta_{1}, \ldots, \beta_{r} \in U_{k}^{D}$. This proves continuity.

Concerning openness, let $k \in \mathbb{N}$. By Remark 4.7, we find $k^{\prime} \in \mathbb{N}$ big enough so that

$$
\phi^{-1} \circ\left(U_{k^{\prime}}^{D}\right)^{r} \subset\left(U_{k}^{D}\right)^{n} \circ \phi^{-1}
$$

Similarly as above, for every $\gamma_{1}, \ldots, \gamma_{n} \in \operatorname{Isom}_{D}(B)$ and $\beta_{1}, \ldots, \beta_{r} \in U_{k^{\prime}}^{D}$, we find $\alpha_{1}, \ldots, \alpha_{n} \in U_{k}^{D}$ such that

$$
\iota\left(\gamma_{1}, \ldots, \gamma_{n}\right)\left(\beta_{1}, \ldots, \beta_{r}\right)=\iota\left(\gamma_{1} \alpha_{1}, \ldots, \gamma_{n} \alpha_{n}\right)
$$

This shows that $\iota$ is open.

Corollary 4.10. $\mathcal{Q}$ is a proper smooth $\mathcal{A}$ - $C W$-complexes.

Proof. This follows from Remark 2.2 together with Remark 4.8, Remark 3.3 and Proposition 4.9

Proposition 4.11. $\mathcal{Q}$ is contractible.

Proof. It suffices to prove that $\mathcal{R}$ is contractible (Proposition 3.2). It is well known that a cofiltered category is contractible. Recall that a category $\mathcal{C}$ is cofiltered if for every two objects $X, Y$ there is another object $Z$ and morphisms from $Z$ to $X$ and $Y$ and if for every pair of morphisms $f_{1}, f_{2}: X \rightarrow Y$ there is a morphism $g: Z \rightarrow X$ with $f_{1} \circ g=f_{2} \circ g$.

We now show that $\mathcal{R}$ is cofiltered. The second property is automatically satisfied since $\mathcal{R}$ is a generalized poset. Let $\phi_{1}, \phi_{2}$ be two objects in $\mathcal{R}$. Since $\phi_{i}: n_{i} B \rightarrow r B$ is a $D$-admissible local similarity, we find a partition $\mathcal{D}_{i}$ of the codomain $r B$ into disjoint balls which are $D$-similar to balls in the domain $n_{i} B$ via $\phi_{i}$. We then find a partition $\mathcal{D}$ of $r B$ into disjoint balls which both refines $\mathcal{D}_{1}$ and $\mathcal{D}_{2}$. Set $n:=|\mathcal{D}|$, i.e. the number of balls in the partition $\mathcal{D}$. Let $\phi: n B \rightarrow r B$ be the unique order-preserving merge map mapping summands in $n B$ to balls in $\mathcal{D}$. Then $\alpha_{i}:=\phi_{i}^{-1} \circ \phi$ are merge maps or transformations and consequently yield arrows $\phi_{1} \leftarrow \phi \rightarrow \phi_{2}$

Proposition 4.12. Each $\mathcal{Q}(k)$ is an $\mathcal{A}$-CW-complex of finite type, i.e. there are only finitely many equivariant cells in each dimension.

Proof. Let $n \in \mathbb{N}$ and $\phi_{1}, \phi_{2}$ be two sphero-vertices of level $n$. Then $\gamma:=\phi_{2} \circ \phi_{1}^{-1}$ is an element in $\mathcal{A}$. Hence in $\mathcal{Q}$ we have $\gamma \cdot\left[\phi_{1}\right]=\left[\phi_{2}\right]$. This proves that any two objects in $\mathcal{Q}$ of the same level are $\mathcal{A}$-equivalent. So there are only finitely many $\mathcal{A}$-classes of objects in $\mathcal{Q}(k)$. In other words, the category $\mathcal{A} \backslash \mathcal{Q}(k)$ has only finitely many objects.

Now let $\left[\phi_{1}\right],\left[\phi_{2}\right]$ be two objects in $\mathcal{Q}$ represented by sphero-vertices $\phi_{i}: n_{i} B \rightarrow$ $r B$. Consider an arrow $\left[\phi_{1}\right] \rightarrow\left[\phi_{2}\right]$ represented by a merge map or a transformation $\alpha$ with $\phi_{2} \circ \alpha=\phi_{1}$. After changing either the representative $\phi_{1}$ or the representative $\phi_{2}$, we may assume that $\alpha$ restricted to any summand $B$ in the domain $n_{1} B$ is 
order-preserving. Observe that there are only finitely many such merge maps or transformations $n_{1} B \rightarrow n_{2} B$ with this property. This observation implies that $\mathcal{Q}(k)$ is locally finite in the sense that any given object is the domain or codomain of only finitely many arrows. Hence also the category $\mathcal{A} \backslash \mathcal{Q}(k)$ is locally finite and thus finite because it has only finitely many objects. So its nerve is of finite type.

4.4. Connnectivity of the descending links. In this subsection, we want to prove that the descending links in $\mathcal{P}$ with respect to the Morse function given by the level of sphero-vertices are highly connected. More precisely: For each $k \in \mathbb{N}$ there is $n \in \mathbb{N}$ such that for all objects $X \in \mathcal{P}$ of Morse height at least $n$ the descending link $l k_{\downarrow}(X)$ is $k$-connected. By Remark 3.7 we get the same result for the descending links of $\mathcal{R}$ and $\mathcal{Q}$. We will be mainly interested in the statement for the generalized poset $\mathcal{Q}$.

Fix $X=[\phi]$ an object in $\mathcal{P}$ of level $n$. The objects of $l k_{\downarrow}(X)$ are in one to one correspondence with merge maps $\mu: n B \rightarrow k B$ with $k<n$ modulo transformations on the codomain, i.e. $\mu$ and $\mu^{\prime}$ are equivalent if and only if there is a transformation $\sigma$ with $\sigma \circ \mu=\mu^{\prime}$. Equivalently, the objects are in one to one correspondence with split maps $\nu: k B \rightarrow n B$ with $k<n$ modulo transformations on the domain. Denote by $\llbracket \nu \rrbracket$ the equivalence class of a split map $\nu$ under this equivalence relation. We have $\llbracket \nu_{1} \rrbracket \rightarrow \llbracket \nu_{2} \rrbracket$ in $l k_{\downarrow}(X)$ if and only if the two objects are equal or if there is a merge map $\mu$ with $\nu_{2} \circ \mu=\nu_{1}$. If such a merge map exists, it exists for any representatives $\nu_{1}, \nu_{2}$ and is uniquely determined by them. Let $l k_{\downarrow}^{*}(X)$ be the subposet spanned by the elements $\llbracket \nu \rrbracket$ with $\nu$ a very elementary split map.

Proposition 4.13. The inclusion $l k_{\downarrow}^{*}(X) \rightarrow l k_{\downarrow}(X)$ is a homotopy equivalence.

Proof. We want to build up $l k_{\downarrow}(X)$ from $l k_{\downarrow}^{*}(X)$ using a Morse function. If $\nu: k B \rightarrow$ $n B$ is a split map which is not very elementary, then we define the Morse height of $\llbracket \nu \rrbracket \in l k_{\downarrow}(X) \backslash l k_{\downarrow}^{*}(X)$ to be the number $n-k$. It suffices to show that the descending link of each such vertex is contractible (and non-empty in particular).

The objects of the descending link $l k_{\downarrow} \llbracket \nu \rrbracket$ are in one to one correspondence with merge maps $\mu$ such that $\nu \circ \mu$ is a split map again, modulo transformations on the domain. Denote the corresponding classes by $\langle\mu\rangle$. We have $\left\langle\mu_{1}\right\rangle \rightarrow\left\langle\mu_{2}\right\rangle$ in $l k_{\downarrow} \llbracket \nu \rrbracket$ if and only if the two objects are equal or if there is a merge map $\rho$ with $\mu_{2} \circ \rho=\mu_{1}$ (which exists independently of the choices of $\mu_{1}, \mu_{2}$ and is uniquely determined by them).

It is now easy to see that the poset $l k_{\downarrow} \llbracket \nu \rrbracket$ is isomorphic to the following poset: As elements we have non-trivial partitions $P$ of $k B$ into disjoint subballs such that the split map $\nu$ either maps balls in $P$ homeomorphically onto summands in $n B$ or splits them even further and such that at least one ball in $P$ is split non-trivially (i.e. $\nu$ maps such a ball homeomorphically onto more than one summand in $n B$ ). We have $P_{1} \geq P_{2}$ if and only if $P_{1}$ is a refinement of $P_{2}$, i.e. if and only if each ball of $P_{1}$ is contained in some ball of $P_{2}$.

Now let $P_{\nu}$ be the partition of $k B$ which divides a summand $B$ into its $q$ maximal proper subballs if and only if $\nu$ splits it non-trivially. Since $\nu$ is assumed to be $n o t$ very elementary, we have $P_{\nu} \in l k_{\downarrow} \llbracket \nu \rrbracket$ and hence $l k_{\downarrow} \llbracket \nu \rrbracket$ is non-empty. Let $P$ be an arbitrary partition in $l k_{\downarrow} \llbracket \nu \rrbracket$. Define $F(P)$ to be the partition which divides a summand $B$ in $k B$ into its $q$ maximal proper subballs if and only if $P$ divides it into subballs. Then we have $P \geq F(P)$. Moreover, $F$ defines an order-preserving map (a functor) $F: l k_{\downarrow} \llbracket \nu \rrbracket \rightarrow l k_{\downarrow} \llbracket \nu \rrbracket$. Last but not least, we have $P_{\nu} \geq F(P)$ for each $P$.

The existence of such an $F$ implies the contractibility of the poset $l k_{\downarrow} \llbracket \nu \rrbracket:$ The arrows $P \geq F(P)$ form a natural transformation from the identity functor of $l k_{\downarrow} \llbracket \nu \rrbracket$ 
to the functor $F$. Furthermore, the arrows $P_{\nu} \geq F(P)$ form a natural transformation from the constant functor with value $P_{\nu}$ to $F$. Natural transformations yield homotopies on the level of spaces. Hence we get a homotopy from the identity to a constant map. Geometrically, $F$ is a deformation retraction into the base of a cone with tip $P_{\nu}$.

It remains to show that the connectivity of the posets $l k_{\downarrow}^{*}(X)$ tends to infinity as $\operatorname{lvl}(X)$ tends to infinity. We first give a simpler description of this poset, starting with the case $D=\operatorname{Sym}(q)$ : Recall that an element in this poset is a class $\llbracket \nu \rrbracket$ of very elementary split maps $\nu: k B \rightarrow n B$ modulo transformations on the domain. In such a very elementary split map, either a summand $B$ of $k B$ is not split at all, or else it is split into its $q$ maximal proper subballs. Thus, such a class $\llbracket \nu \rrbracket$ is already uniquely determined by the information which summands in $n B$ arise from a splitting of a single summand in $k B$. So the elements in the poset $l k_{\downarrow}^{*}(v)$ can be regarded as partitions $P$ of the set $\mathbf{n}=\{1, \ldots, n\}$ into subsets of cardinality either 1 or $q$ (the elements in $\mathbf{n}$ represent the summands in $n B$ ) and there has to be at least one subset of cardinality $q$. We have $P_{1} \geq P_{2}$ if and only if $P_{1}$ is a refinement of $P_{2}$.

Now consider the case $D=\{1\}$ : The main difference to the discussion above is that whenever a summand of $k B$ is split by $\nu$ into its $q$ maximal proper subballs, we have to remember which summands of $n B$ correspond to which of the $q$ maximal proper subballs. In the point of view of partitions from above, we can model this information by decorating each subset of cardinality $q$ in a partition of $\mathbf{n}$ representing an object in $l k_{\downarrow}^{*}(X)$ with an element in $\operatorname{Sym}(q)$. In the general case where $D$ is an arbitrary subgroup of $\operatorname{Sym}(q)$, the decorations are elements in the set of cosets $\operatorname{Sym}(q) / D$.

We can simplify the description even more: The geometric realization of this poset is the barycentric subdivision of the following flag complex $\mathcal{C}_{n}$ : As vertices we have subsets $x$ of $\mathbf{n}$ of cardinality $q$ together with a decoration in $\operatorname{Sym}(q) / D$. We join two such vertices $x_{1}, x_{2}$ by an edge if and only if they are disjoint as subsets of $\mathbf{n}$. The following proposition concludes the proof of the connectivity claim.

Proposition 4.14. A lower bound for the connectivity of $\mathcal{C}_{n}$ is given by the formula

$$
\nu(n)=\left\lfloor\frac{n-q}{2 q-1}\right\rfloor-1
$$

Proof. This is a special case of [22, Theorem 4.7]: Consider a single color $*$ and for each element in $\operatorname{Sym}(q) / D$ an archetype of length $q$. Then we have

$$
\mathcal{C}_{n}=\mathcal{A C}_{3}(\{*\}, \operatorname{Sym}(q) / D ; \mathbf{n})
$$

Since $m_{a}=q=m_{r}$, the formula follows.

We provide the proof for the convenience of the reader. It is an induction over $n$. The induction start is $n \geq q$ because $q$ is the first number such that $\nu(q) \geq-1$. Indeed, it is obvious that $\mathcal{C}_{n}$ is not empty in the case $n \geq q$.

For the induction step, let $b$ be the vertex $\{1, \ldots, q\} \subset \mathbf{n}$ with arbitrary decoration. Consider the full subcomplex $\mathcal{C}_{n}^{\prime}$ spanned by the vertices which are disjoint from $b$. The first step is to estimate the connectivity of the pair $\left(\mathcal{C}_{n}, \mathcal{C}_{n}^{\prime}\right)$ using the discrete Morse technique for simplicial complexes. Let $a$ be a vertex in $\mathcal{C}_{n} \backslash \mathcal{C}_{n}^{\prime}$. In other words, $a$ intersects $b$ non-trivially. Consider the binary number $f(a)$ with $q$ digits such that the $i$ 'th digit is 0 whenever $i \notin a$ and 1 whenever $i \in a$. When ordering this binary numbers in the natural way, $f$ becomes a Morse function building up $\mathcal{C}_{n}$ from $\mathcal{C}_{n}^{\prime}$.

We need to estimate the connectivity of the descending link $l k_{\downarrow}(a)$. It is the full subcomplex of $\mathcal{C}_{n}$ spanned by the vertices $x$ which are disjoint from $a$ and such that 
$\min a<\min x$. Thus we see that it is isomorphic to the complex $\mathcal{C}_{k}$ with

$$
k=n-q-(\min a-1) \geq n-q-q+1=n-(2 q-1) .
$$

It follows, by induction, that $l k_{\downarrow}(a)$ is $\nu(n-(2 q-1))$-connected. Hence, the connectivity of the pair $\left(\mathcal{C}_{n}, \mathcal{C}_{n}^{\prime}\right)$ is $\nu(n-(2 q-1))+1=\nu(n)$.

In the last step, we observe that the inclusion $\iota: \mathcal{C}_{n}^{\prime} \rightarrow \mathcal{C}_{n}$ induces the trivial map in $\pi_{m}$ for $m \leq \nu(n)$. It then follows from the long exact homotopy sequence that $\mathcal{C}_{n}$ is $\nu(n)$-connected. So let $\varphi: S^{m} \rightarrow \mathcal{C}_{n}^{\prime}$ be a map which we can assume to be simplicial by simplicial approximation. Then we have $\operatorname{im}(\iota \circ \varphi) \subset \operatorname{star}(b)$ and $\iota \circ \varphi$ can be homotoped within the star of $b$ to the constant map with value the vertex $b$.

4.5. Conclusion of the proof of Theorem 1.4. Consider the contractible proper smooth $\mathcal{A}_{q r}^{D}$-CW-complex $\mathcal{Q}$ (Corollary 4.10 and Proposition 4.11). It is not yet of finite type. However, we claim that the filtration $\mathcal{Q}(k)$ is of finite type and $\mathscr{C} \mathscr{O}_{-}$ highly connected where $\mathscr{C} \mathscr{O}$ is the family of compact open subgroups of $\mathcal{A}_{q r}^{D}$. We can then apply Theorem 2.9 and the proof is complete.

That each $\mathcal{Q}(k)$ is of finite type follows from Proposition 4.12, For the second statement, observe that whenever $X$ is an object in $\mathcal{Q}^{H}$ (compare with Remark 3.4) with $H \in \mathscr{C} \mathscr{O}$ and $Y \in \mathcal{Q}$ with $X \rightarrow Y$, then also $Y \in \mathcal{Q}^{H}$ : Because let $X=[\phi]$, then for each $\gamma \in H$ the map $\phi^{-1} \circ \gamma \circ \phi$ is a strict transformation. If $Y=[\psi]$ then there is a merge map $\mu$ with $\psi \circ \mu=\phi$. Since for each strict transformation $\nu$ such that $\mu \circ \nu$ is defined we have that $\mu \circ \nu \circ \mu^{-1}$ is again a strict transformation, we see that $\psi^{-1} \circ \gamma \circ \psi$ is a strict transformation for every $\gamma \in H$. In other words, $Y$ is fixed by $H$.

It follows from the above observation that a descending link $l k_{\downarrow}^{H}(X)$ with respect to the filtration $\mathcal{Q}(k)^{H}$ of $\mathcal{Q}^{H}$ is equal to the descending link $l k_{\downarrow}(X)$ with respect to the filtration $\mathcal{Q}(k)$ of $\mathcal{Q}$. In Subsection 4.4 we have shown that the latter are highly connected. Hence also the descending links $l k_{\downarrow}^{H}(X)$ are highly connected, uniformly in $H$. This implies that the filtration $\mathcal{Q}(k)$ is $\mathscr{C} \mathscr{O}$-highly connected and finishes the proof of Theorem 1.4.

\section{REFERENCES}

[1] H. Abels and A. Tiemeyer, Compactness properties of locally compact groups, Transform. Groups 2 (1997), no. 2, 119-135.

[2] U. Bader, P.-E. Caprace, T. Gelander, and S. Mozes, Simple groups without lattices, Bull. Lond. Math. Soc. 44 (2012), no. 1, 55-67.

[3] G. E. Bredon, Topology and geometry, Graduate Texts in Mathematics, vol. 139, SpringerVerlag, New York, 1997. Corrected third printing of the 1993 original.

[4] M. R. Bridson and A. Haefliger, Metric spaces of non-positive curvature, Grundlehren der Mathematischen Wissenschaften [Fundamental Principles of Mathematical Sciences], vol. 319, Springer-Verlag, Berlin, 1999.

[5] K. S. Brown, Cohomology of groups, Graduate Texts in Mathematics, vol. 87, Springer-Verlag, New York, 1994. Corrected reprint of the 1982 original.

[6] _ Finiteness properties of groups, Proceedings of the Northwestern conference on cohomology of groups (Evanston, Ill., 1985), 1987, pp. 45-75.

[7] K. S. Brown and R. Geoghegan, An infinite-dimensional torsion-free $\mathrm{FP}_{\infty}$ group, Invent. Math. 77 (1984), no. 2, 367-381.

[8] P.-E. Caprace and T. De Medts, Simple locally compact groups acting on trees and their germs of automorphisms, Transform. Groups 16 (2011), no. 2, 375-411.

[9] I. Castallano and T. Weigel, Rational discrete cohomology for totally disconnected locally compact groups (2015). ArXiv e-prints 1503.02436.

[10] Y. de Cornulier and P. de la Harpe, Metric geometry of locally compact groups, 2015. ArXiv e-prints 1403.3796

[11] R. Geoghegan, Topological methods in group theory, Graduate Texts in Mathematics, vol. 243, Springer, New York, 2008. 
[12] B. Hughes, Trees and ultrametric spaces: a categorical equivalence, Adv. Math. 189 (2004), no. 1, 148-191.

[13] C. Kapoudjian, From symmetries of the modular tower of genus zero real stable curves to a Euler class for the dyadic circle, Compositio Math. 137 (2003), no. 1, 49-73.

[14] _ Simplicity of Neretin's group of spheromorphisms, Ann. Inst. Fourier (Grenoble) 49 (1999), no. 4, 1225-1240.

[15] M. Kneser, Erzeugende und Relationen verallgemeinerter Einheitengruppen, J. Reine Angew. Math. 214/215 (1964), 345-349 (German).

[16] A. Le Boudec, Compact presentability of tree almost automorphism groups (2014). ArXiv e-prints 1402.5652 .

[17] W. Lück, Survey on classifying spaces for families of subgroups, Infinite groups: geometric, combinatorial and dynamical aspects, Progr. Math., vol. 248, Birkhäuser, Basel, 2005, pp. 269-322.

[18] , The type of the classifying space for a family of subgroups, J. Pure Appl. Algebra 149 (2000), no. 2, 177-203.

[19] - Transformation groups and algebraic K-theory, Lecture Notes in Mathematics, vol. 1408, Springer-Verlag, Berlin, 1989. Mathematica Gottingensis.

[20] N. E. Steenrod, A convenient category of topological spaces, Michigan Math. J. 14 (1967), $133-152$.

[21] M. Stein, Groups of piecewise linear homeomorphisms, Trans. Amer. Math. Soc. 332 (1992), no. 2, 477-514.

[22] W. Thumann, Operad groups and their finiteness properties (2015). ArXiv e-prints 1409.1085.

[23] T. tom Dieck, Transformation groups, de Gruyter Studies in Mathematics, vol. 8, Walter de Gruyter \& Co., Berlin, 1987.

[24] S. Waner, Equivariant homotopy theory and Milnor's theorem, Trans. Amer. Math. Soc. 258 (1980), no. 2, 351-368.

Karlsruhe Institute of Technology, Karlsruhe, Germany

E-mail address: roman.sauer@kit.edu

Max Planck Institute for Mathematics, Bonn, Germany

E-mail address: thumann@math.uni-bonn.de 